MATEC Web of Conferences 33, 03015 (2015)

DOI: $10.1051 /$ matecconf/ 20153303015

(C) Owned by the authors, published by EDP Sciences, 2015

\title{
Special cases of martensite compatibility: A near single-variant habit- plane and the martensite of nanocrystalline NiTi
}

\author{
Manuel Petersmann ${ }^{1, a}$, Thomas Antretter ${ }^{1}$ and Thomas Waitz ${ }^{2}$ \\ ${ }^{1}$ Institute of Mechanics, Montanuniversitaet Leoben, Franz-Josef-Straße 18, 8700 Leoben, Austria \\ ${ }^{2}$ Physics of Nanostructured Materials, Faculty of Physics, University of Vienna, Boltzmangasse 5, 1090 Vienna, Austria
}

\begin{abstract}
Lattice parameters measured near the high temperature $\left(\sim 1000^{\circ} \mathrm{C}\right)$ bcc $\alpha$ to hcp $\beta$ transformation in an intermetallic Mo-containing $\gamma$-TiAl based alloy indicate a middle valued eigenvalue of the corresponding deformation gradient near 1. Habit-planes calculated under the assumption of a simple slip as lattice invariant shear, agree with experimentally determined orientations of the lens like plates recorded via electron backscattering. By contrast, twinning as invariant lattice shear has been investigated in nanocrystalline NiTi. Here the grain size causes the formation mechanism of the martensite to change from a "herring-bone" morphology faciliting a habit-plane between two twinned laminates and the austenite to a single laminate, which in the nonlinear theory formally cannot form a habit-plane with the austenite. Since this might cause high accommodation strains, the effectiveness of stress accommodation of martensite formed in neighboring grains of a polycrystal is investigated. Subsequent numerical microstructural modeling is outlined. The resulting energetically most favorable transformation sequence yields the transformation kinetics.
\end{abstract}

\section{Introduction}

Martensitic transformations are accompanied by two types of inhomogeneous, lattice invariant deformation mechanisms, namely slip or twinning, as is illustrated in Fig 1. The habit-plane defined by its normal vector $\mathbf{h}$, is separating austenite from martensite (shaded grey in Fig.1). Since the inhomogeneous lattice invariant deformation causes the plane to be tilted off its original orientation, the habit-plane of martensite in general has no integer indices. Particularly, at the atomic level there is no invariant plane, but a narrow flat transition zone. The type of accommodation mechanism mainly depends on the material. Recently, the subject of reversibility of the transformation has been discussed to depend on the lattice symmetry [1]. Additionally the grain size, temperature, strain rate, etc. play a major role. Particularly, the temperature and strain rate dependence of twinning is comparatively small compared to that of slip, which is highly facilitated at higher temperatures and lower strain rates. Conventional steels for instance show local plastic flow for bainite and twinning for "classical" martensite at higher cooling rates [2]. Recently, the interaction of twinning and slip also has been discussed in high temperature shape memory alloys [3]. In the present work one dominating lattice invariant deformation mechanism is considered. Furthermore, the simplest forms of mechanisms for invariant planes are considered, i.e. simple shear (as opposed to works considering double or multiple shears). Twinned martensite might form as a single laminate of alternating twins, or as a more complex hierarchic twin laminate structure, see e.g. [4].

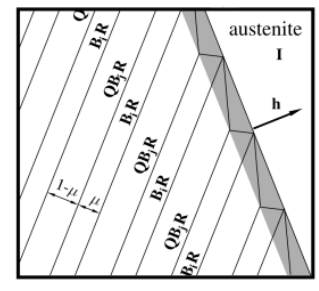

twinned martensite laminate

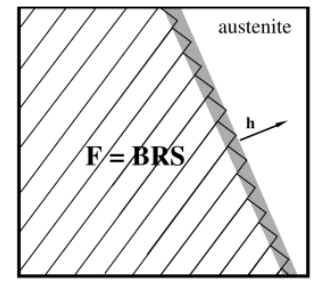

slipped martensite matching macroscopic shape and observed structure

Fig.1: Illustration of the phenomenological theory of martensite crystallography. To obtain the valid lattice, orientations relation and shape a lattice invariant deformation in form of slipping or twinning has to be imposed.

\subsection{Common features}

Martensite is characterised by the formation of symmetry related, Bain correspondence variants (BCVs). The number of BCVs depends on the point group symmetries of the parent and product phases. Let the number of point group symmetries of the austenite lattice be $\# \mathcal{P}^{\mathrm{a}}$ and that of the martensite $\# \boldsymbol{P}^{\mathrm{m}}$. Frequently the symmetry of the austenite is greater than that of the martensite (i.e. $\# \boldsymbol{P}^{\mathrm{a}}>$ $\# \mathcal{P}^{\mathrm{m}}$ ) and the point groups of the austenite and martensite show a group/subgroup relation. Then the number of BCVs is given by $\# \boldsymbol{P}^{\mathrm{a}} / \# \boldsymbol{P}^{\mathrm{m}}$. Note, that this condition does not apply to a cubic austenite and a hexagonal

\footnotetext{
${ }^{\mathrm{a}}$ Corresponding author: manuel.petersmann@unileoben.ac.at
} 
martensite as will be elaborated in section 3. There might be more deformation gradients $\mathbf{F}$ than Bain-strains $\mathbf{B}$ for the martensite, since some Bain-strains coincide because the deformation gradients differ only by their rotational parts. Assuming $\mathbf{F}=\mathbf{B R}, \mathbf{B}$ and $\mathbf{R}$ can easily be calculated, since $\mathbf{F} \mathbf{F}^{\mathbf{T}}=\mathbf{B} \mathbf{R} \mathbf{R}^{\mathbf{T}} \mathbf{B}^{\mathbf{T}}=\mathbf{B}^{\mathbf{2}}$. Hence $\mathbf{B}=\sqrt{\mathbf{F F}^{\mathbf{T}}}$ and $\mathbf{R}=\mathbf{F B}^{\mathbf{- 1}}$. In order to find an invariant line the squared eigenvalues $\lambda_{i}^{2}$ of $\mathbf{B}^{2}$ (directly yielding $\lambda_{i}$ ) have to be calculated. A necessary and sufficient condition for an invariant plane to exist is that one of the eigenvalues of $\mathbf{B}$ must be $>1$, another $<1$ and the intermediate value $=1$, or if the eigenvalues are arranged in ascending order $[5,6]$ :

$$
\lambda_{1}<1, \quad \lambda_{2}=1, \quad \lambda_{3}>1
$$

$\lambda_{2}=1$ is also referred to as the first cofactor conditions [7, 8]. If these conditions are fulfilled, then an invariant plane is possible for every choice of $\mathbf{R}$. The above and two other cofactor conditions [7,8] are necessary and sufficient for the equations of the crystallographic theory of martensite to be satisfied for a given twin system, but for any twin-volume fraction $\mu$ : $0 \leq \mu \leq 1$, implying a low hysteresis and improved resistance to functional fatigue [7-10]. In general these conditions are not fulfilled and only two solutions for $\mu$ and $1-\mu$ are obtained [7-10]. The most general form of an invariant plane strain (IPS) $\mathbf{P}$ can be written as $[2,11]$ :

$$
\mathbf{P}=\mathbf{I}+\mathbf{d} \otimes \mathbf{h}=\mathbf{F R}_{\mathbf{I}}
$$

Where I denotes the unity matrix, $\mathbf{h}$ is the normal vector of the invariant plane and the vector $\mathbf{d}$ describes the displacement of a point $\mathbf{x}$ under the deformation $\mathbf{P}$. see Fig.1. There are formulae for the calculation of $\mathbf{d}$ and $\mathbf{h}$ from the eigenvalues and eigenvectors of $\mathbf{F}$ see e.g. $[5,6,11]$.

\section{Examples}

\subsection{Near perfect single-variant habit-plane}

The lattice correspondence between the parent $\beta$ - and the product $\alpha$-phase in Ti-Al-Mo is given by the Burgers orientation relation (OR) [13]. The Bain-strain is most conveniently formulated in a Cartesian CS, where two vectors lie in an $(011)_{\beta}$ plane, e.g. X $\left\|[10 \overline{1}]_{\beta}, Y\right\|$ $[010]_{\beta}, Z \|[101]_{\beta}$. After the transformation the $(0001)_{\alpha}$ basis plane of a $\mathrm{BCV}$ corresponds to one of the 6 possible $(011)_{\beta}$ planes. A $\langle 11 \overline{2} 0\rangle_{\alpha}$ direction of each BCV corresponds to one out of two possible $<111>_{\beta}$ lattice vectors of the parent phase. However, these BCV only differ by a rotation around their c-axis by a small angle, see $[12,13]$. Therefore, the six $\langle 011\rangle_{\beta}$ planes correspond to six Bain-strains. The two possible rotations for each Bain-strain $\mathbf{B}$ result in 12 distinct deformation gradients F. However, in any case $\# \boldsymbol{P}^{\mathbf{a}} / \# \boldsymbol{P}^{\mathbf{m}}=2 \neq 6$ as mentioned above.

In general, the lattice parameters measured near the transformation temperature yield an intermediate eigenvalue close to but not exactly 1 . As already pointed out this has major consequences for the material behavior [9-10]. An accurate agreement of $\lambda_{2}=1$ can be achieved by superimposing a simple shear on the deformation representing plastic slip.

$$
\mathbf{F}=\mathbf{B R S}
$$

From the physical point of view this represents an inhomogeneous deformation. Note, that theoretically a single BCV can always be superimposed with a homogeneous shear in a proper coordinate system so that an invariant plane can be formed. However, it remains to be verified that the slip system as well as the shearmagnitude are physically reasonable. $\mathbf{S}$ contains the shear magnitude that needs to be adjusted such that the solution of the following eigenvalue problem

$$
\operatorname{det}\left(\mathbf{S}^{\mathbf{T}} \mathbf{B}^{\mathbf{T}} \mathbf{B S}-\lambda^{2} \mathbf{I}\right)=0
$$

yields $\lambda_{2}=1$. Eq. 4 has either one real algebraic root in the case of a simple shear, i.e. $\mathbf{B}=\mathbf{I}$ or three. From Eq. 4 the shear magnitude must then be evaluated for all physically distinct slip systems and each BCV. We investigated a bcc-hcp transformation (see conclusions), where it turns out that there are two slip systems with equal minimal shear-magnitude for each BCV with specific $\{112\}_{\beta}$ slip plane and $\langle 111\rangle_{\beta}$ shear direction [12]. Note that this result agrees with the observation that that Burgers orientation relation renders the $(112)_{\beta}$ plane nearly parallel to the $(1 \overline{1} 00)_{\alpha}$ plane [13]. The second lowest value of the possible solutions for the required shearmagnitude is already $15 \%$ higher. Even if other slip modes may appear, it is reasonable to assume that the shear system yielding the lowest shear amplitude is the most favorable one and will thus be realized in nature. Since two shear systems have been found for each $\mathbf{B}_{\mathbf{i}}$ requiring the calculated minimum shear there are 12 modified Bain-strains $\overline{\mathbf{B}}_{\mathbf{i}}^{\mathbf{j}}=\mathbf{S}_{\mathbf{j}} \mathbf{B}_{\mathbf{i}}$ that can be used for the calculation of invariant habit-planes yielding 24 habit planes because of the alternating sign in the solution of Eq.2 [5,6,15].

\subsection{Compound twinning in polycrystalline nanocrystalline equiatiomic $\mathrm{NiTi}$}

In "coarse-grained" (grain size $>1 \mu \mathrm{m}$ ) NiTi the shape change of the martensite is accommodated by various types of twins. Beside type I (mirror or equivalently, reflection symmetry at twin plane $\mathrm{K}_{1}$ ) and type II (twofold rotational symmetry around the shear direction) twins, both also often referred as conventional twins [5], also so-called non-generic twins [16] are found. Generic twins are transformation twins that can form for any lattice parameters in the martensite and are thus purely "symmetry-driven". By contrast, the existence of nongeneric twins is restricted to particular lattice parameters [18]. A twin showing both, reflection and mirror symmetry at the twin plane, is termed compound twin. The twinning mechanism for the most common formation of a plate like morphology with an invariant plane is illustrated in Fig. 1 on the left side. Two distinct variants 
coherently join, forming a laminate plate, which in turn forms an invariant plane generally accompanied by an elastic transition layer with the austenite, where the width of the transition layer depends on the lattice parameters [7]. Since twinned martensite requires no irreversible plastic slip for the formation of an invariant plane, only the "pure"/symmetric part of the deformation gradient obtained from the polar decomposition needs to be considered in the construction of microstructures of shape-memory alloys [5]. For the BCVs of the laminate the compatibility condition reads:

$$
\mathbf{R B}_{\mathbf{j}}-\mathbf{B}_{\mathbf{i}}=\mathbf{a} \otimes \mathbf{n}
$$

Here $\mathbf{n}$ is the normal vector of the plane twin interface and the vector $\mathbf{a}$ is similar to $\mathbf{d}$ in Eq.1. This equation also is referred to as "twinning equation". Note the exact same structure as in Eq.2. If Eq.8 is post-multiplied by $\mathbf{B}_{\mathbf{i}}$ and each side is multiplied by its transpose a symmetric matrix $\mathbf{C}_{\mathbf{t}}=\mathbf{B}_{\mathrm{i}}^{-1} \mathbf{B}_{\mathrm{j}}{ }^{2} \mathbf{B}_{\mathrm{i}}^{-1}$ is obtained, whose eigenvalues must satisfy Eq. 1 in order to form a twin. Also the vectors $\mathbf{a}$ and $\mathbf{n}$ are calculated in analogy to $\mathbf{d}$ and $\mathbf{h}$ in Eq.2, then $\mathbf{R}$ is obtained by rearranging Eq.8. The solutions of the twinning equation come in pairs. The two different twins obtained from a solution are called reciprocal twins. If two compatible $\mathrm{BCVs}$ are identified the average deformation of the laminate is given by

$$
\mathbf{F}_{\boldsymbol{\mu}}=\mathrm{Q}\left\{\mu \mathbf{R} \mathbf{B}_{\mathbf{j}}+(1-\mu) \mathbf{B}_{\mathbf{i}}\right\}
$$

Where $\mathbf{Q}$ is an additional rotation and $\mu$ is the BCV fraction, see Fig.1. Then the IPS matrix $\mathbf{P}$ can be found from Eq.2 by substituting $\boldsymbol{F}_{\boldsymbol{\mu}}$ for $\mathbf{F}$. The laminate plate averages the misfit of the individual $\mathrm{BCVs}$ to produce an overall invariant fit. The interfacial strains of the individual $\mathrm{BCV}$ are local strains with no long range consequences, so that they can be treated as a surface energy $\Gamma_{t w}$ [17]. Also at this point the mathematical equivalence to the slipped formalism has been shown $[15,18]$.

For nanocrystalline $\mathrm{NiTi}$, it has been reported that the martensite start temperature $\mathrm{M}_{\mathrm{S}}$ is significantly lower than in the course-grained material $[19,20]$. Evidently, the energy-barrier for the onset of martensite formation is considerably size-dependent, increasing with decreasing grain size, where a general suppression of thermally induced B19' martensite below a critical grain size of equivalent sphere diameter of about $50 \mathrm{~nm}$ is reported [19]. Also the temperature interval of the transformation in nanocrystalline NiTi is broader than in its coarsegrained counterpart, indicating that the accommodation of transformation strains is less effective than in larger grains [20]. In nanocrystalline NiTi the grain boundaries impose severe geometrical constraints on the formation of martensite. Experimentally, mainly compound twin laminates of two unique BCVs (out of twelve) denoted as pairings 1-1', 2-2',...6-6' [19], are observed, because they average out the overall shear deformation most effectively, therefore providing the best accommodation mechanism at this scale. Nevertheless, the overall deformation of the laminate still contains a considerable amount of shear. Lamella widths of the BCVs decreases with the grain size [17] and BCVs down to a width as low as $0.9 \mathrm{~nm}$ have been observed via TEM, approximately corresponding to a stack of 4 atomic planes. Dislocations are only rarely observed; hence plastic deformation virtually plays no role [19]. In the absence of an external stress field the laminates consist on average of equal amounts of the corresponding BCVs $(\mu=0.5)$, causing the lowest increase of strain energy. Therefore the deformation of each grain after transformation can be approximated by $\mathbf{F}_{\mathbf{m}}=1 / 2\left(\mathbf{B}_{\mathbf{i}}+\mathbf{R}_{\mathbf{j}} \mathbf{B}_{\mathbf{k}}\right)=1 / 2\left(\mathbf{F}_{\mathbf{i}}+\mathbf{F}_{\mathbf{i}}{ }^{\prime}\right)$, $\mathrm{i}, \mathrm{j}, \mathrm{k}=1 \ldots 6$. Note that the formation of invariant habitplanes is not possible with the formalism presented here for the occurring compound twin type in nanocrystalline NiTi [22]. Therefore the formation suggests very high accommodation strains. Hence the effectiveness of accommodation in the polycrystal aggregate remains to be investigated.

In this special case we used the fact that under a certain grain-size only 6 different laminates are observed, which allows setting up a numerical model to study the effectiveness of accommodation more systematically. Particularly, an incremental energy minimising algorithm has been programmed, automatically searching for a minimum increase in free energy in all possible transformations of one further martensite grain in a $3 \mathrm{D}$ polycrystalline, finite element RVE model consisting of 180 grains visualized in Fig. 2. So to find the energetically favourable transformation for the first grain, $180 \times 6$ finite element caluclations have to be carried out. For the second $179 \times 6$ and so on until for the last grain 6 calculations are necessary to find the best option.

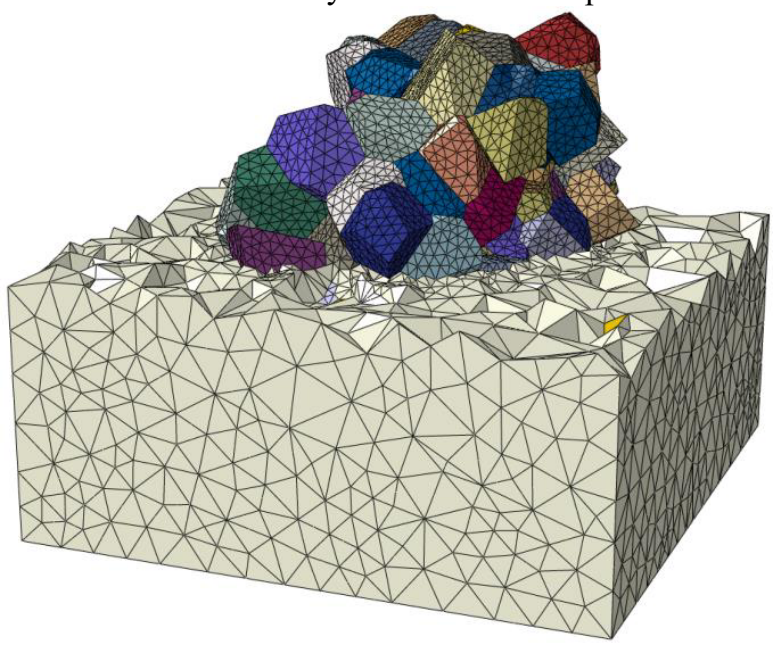

Fig.2: Polycrystalline finite element RVE (colored, finely meshed Voronoi tessellations) embedded in a cubic matrix with a biased mesh.

Beside the strain energy, interface energies make up a significant part of the free energy due to the small volume to surface ratio at this scale. The total difference in free energy thus is

$$
\left\{\Delta \varphi_{i, j}\right\}_{\text {min }}=\left\{\Gamma_{i n}+\Gamma_{t w}+\Sigma_{w}+f_{c}+\frac{U_{i}}{V_{i}}\right\}_{\text {min }}-\int s d T
$$

Where $\Gamma_{i n}, \Sigma_{w}, f$ are the IE between the twins, the IE between austenite and martensite and the work of friction of the transformation [17]. As boundary condition for the 
RVE a self-consistent scheme has been applied, where the matrix surrounding the RVE has the averaged elastic properties of the RVE [18]. A coarser mesh has been applied to find the transformation sequence of the grains in the RVE. This sequence has been recalculated with a fine mesh. Anisotropic elastic constans from ab initio calculations have been used in the calculations [21,22]. In Fig. 3 the calculated minimum specific transformation barriers $\mathrm{e}_{\mathrm{b}}$ and their compositions for each transforming increment of the transformation, i.e. for each martensite fraction in terms of the transformed grain number are visualized. It can be seen that i) the strain energy dominates the transformation energy and ii) the contribution of the strain energy to the energy barrier increases on average as the transformation proceeds making the forming stress field mainly responsible for the transformation rate to decrease as the transformation proceeds.

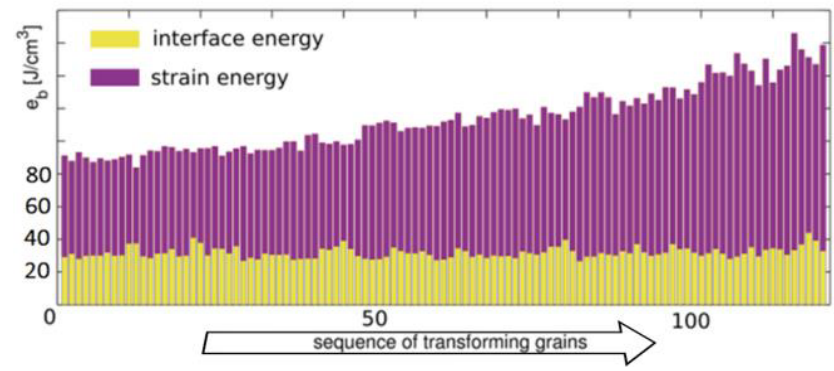

Fig.3: Sequence of subsequent specific transformation barriers for the transformation of a martensite grain. The strain energy part of the barrier increases on average as the transformation proceeds.

Alternatively, the total change of the free energy due to the martensite transformation of the whole RVE can be related to the martensite volume fraction. It is then found that the experimentally measured value for the reversible strain energy is significantly lower than the calculated one. One reason is that the measurement is dominated by larger grains showing other morphologies than in the simulation. Particularly two laminates sharing an invariant plane within one grain, i.e. a junction plane. This morphology is also confirmed by crystallographic calculations [19]. The number of junction planes increases the larger the grain size becomes, accommodating the more efficiently the a.m. remaining overall shear of the laminate. Such a pattern, found in larger grains, can be classified as the well-known herringbone morphology. An exemplary herringbone morphology is given in Fig.4.a. Since the experimental measurements are dominated by the larger grains transforming first the potential of the herringbone morphology has been investigated in a 3D finite element RVE model, where the twin laminates have been resolved, see Fig.4. The parameter d, the twin width and the middle plate width $B$ have been investigated. It turns out that the optimal twin width increases from slightly above $1 \mathrm{~nm}$ for a grain size of about $50 \mathrm{~nm}$ to $2 \mathrm{~nm}$ for a grain size of around $100 \mathrm{~nm}$ equivalent sphere size and the optimum middle plate width is about $30 \%$ of the diameter. With decreasing grain size the single laminate structure at $50 \mathrm{~nm}$ sphere diameter becomes more favourable from an energetic point of view [23]. It should be noted however, that the calculations in [23] were carried out with isotropic macroscopic values for NiTi from the literature rather than anisotropic elastic constants from ab initio calculations.

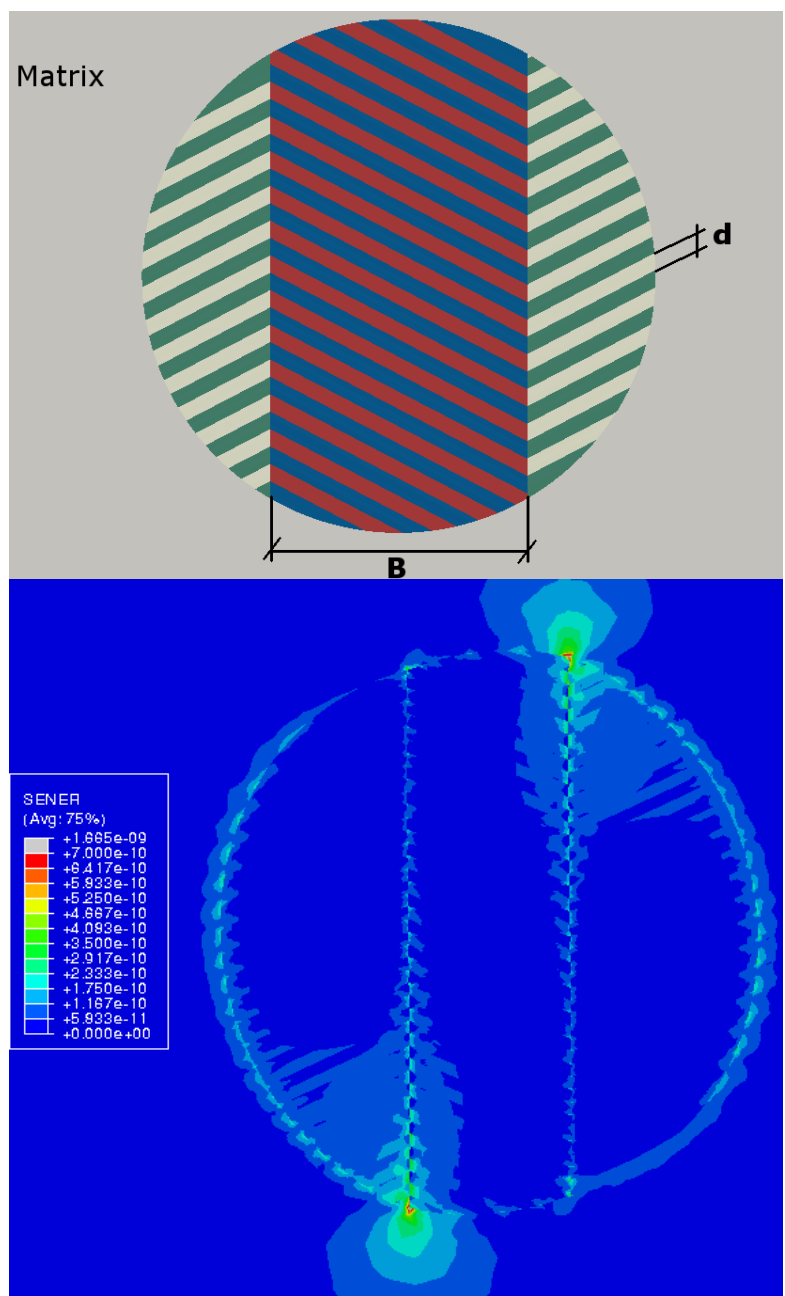

Fig.4: a) Illustration of herringbone morphology in a spherical grain. Two laminates of two martensite variants respectively form an invariant junction plane. $\mathrm{B}$ is the width of the middle laminate. b) Strain energy distribution for B equal to $30 \%$ of the sphere's diameter.

\section{Conclusions}

Since only a small shear strain is necessary for obtaining $\lambda_{2}=1$, a single variant martensite to austenite habit-plane is possible in a Mo-containing gamma-TiAl based alloy [12], a promising material for aerospace applications. The formation of compound-twinned B19' martensite, not facilitating an invariant habit plane in nanocrystalline $\mathrm{NiTi}$, has been studied numerically, using a polycrystal model with averaged grain properties, as well as a single grain model, with detailed microstructure to quantify accommodation mechanisms at both levels. 


\section{Acknowledgement}

Experimental data regarding the Molybdenum stabilized TiAl alloy provided by the department of Physical Metallurgy and Materials Testing at the Montanuniversitaet Leoben and financial support by the Austrian Federal Government (in particular from Bundesministerium für Verkehr, Innovation und Technologie and Bundesministerium für Wissenschaft, Forschung und Wirtschaft) represented by Österreichische Forschungsförderungsgesellschaft $\mathrm{mbH}$ and the Styrian and the Tyrolean Provincial Government, represented by Steirische Wirtschaftsförderungsgesellschaft $\mathrm{mbH}$ and Standortagentur Tirol, within the framework of the COMET Funding Programme is gratefully acknowledged.

\section{References}

1. K. Bhattacharya, S. Conti, G. Zanzotto, Crystal Letters to Nature, 428: 55-59 (2004)

2. H. K. D. Bhadeshia, Bainite in steels, Institute of Materials, London U.K. (1992)

3. J. Ma, I. Karaman, R. D. Noebe, Int. Mat. Reviews 55: 257-315 (2010)

4. H. Seiner, L. Straka, O. Heczko. J. Mech. Phys. Solids. 64 198-211 (2014)

5. K. Bhattacharya. Microstructure of martensite. University Press, Oxford (2003)

6. J. Ball, R. James, Arch. Ration. Mech, 100:13-52 (1987)

7. X. Chen, V. Srivastava, V. Dabade, and R.D. James. J. Mechan. Phys. Solids, 61:2566-2587 (2013)

8. Z. Zhang, Special lattice parameters and the design of low hysteresis materials, Phd Thesis, University of Minnesota, 2007

9. R. Delville, D. Schryvers, Z. Zhang, R.D. James, Scripta Materialia, Elsevier, 2009, 60, 293-296

10. Y. Song, X. Chen, V. Dabade, T.W. Shield, R.D. James, Nature, 502:85-88 (2013)

11. L. Qi, A. Khachaturyan, J. W. Morris Jr., Acta Mater, 76:23 - 39, (2014)

12. S. Mayer, M. Petersmann, (2015) Experimental and theoretical evidence of displacive martensite in an intermetallic Mo-containing $\gamma$-TiAl based alloy, Manuscript submitted for publication.

13. S. Banerjee, P. Mukhopadhyay. Phase Transformations: Examples from Titanium and Zirconium Alloys. Materials Series, Elsevier; (2007)

14. H.M. Otte, R.I. Jaffee, N.E. Promisel, Proc. 1st Int. Conf. Titanium. Oxford: Pergamon Press, (1996)

15. R.D. James and K.F. Hane. Acta Mater, 48:197-222, (2000)

16. M. Pitteri, G. Zanzotto. Acta Mater, 46:225-237 (1996)

17. T. Waitz, T. Antretter, F.D. Fischer, N.K. Simha, and H.P. Karnthaler. J. Mech. Phys. Solids, 55:419-444 (2007)

18. K.F. Hane and T.W. Shield. Acta Mater, 47:26032617 (1999)

19. T. Waitz. Acta Mater, 53:2273-2283 (2005)
20. M. Peterlechner, T. Waitz, C. Gammer, T. Antretter, Int. J. Mater. Res., 6:102, (2011)

21. R. Golesorktabar, Ab initio calculation of elastic properties; General implementation and specific application to the shape-memory material NiTi. Phd Thesis, University of Leoben (2013)

22. M. Wagner, W. Windl. Acta Mater, 56:59-60 (2008)

23. T. Waitz, W. Pranger, T. Antretter, F.D. Fischer, H.P. Karnthaler, Mater. Sci. Eng. A 481-482:479483 (2008) 
